\title{
Sporadic Pediatric GIST With Novel Gene Mutation
}

\author{
Shagufta Jabeen ${ }^{\mathrm{a}, \mathrm{d}}$, Jagannathan Krishnasamy ${ }^{\mathrm{b}}$, Sohail Iqbal ${ }^{\mathrm{c}}$, \\ Andrew Fagbemi ${ }^{\mathrm{b}}$, Ross J Craigie ${ }^{\mathrm{a}}$
}

\begin{abstract}
Advanced understanding of pathogenesis and tumor metabolism has increased the number of recently diagnosed gastrointestinal stromal tumors. We present a case of a young girl who attended our hospital with an episode of melena. Endoscopy revealed an irregular gastric lump with overlying ulceration. Following further investigation and biopsy, a gastrointestinal stromal tumor of the stomach was diagnosed and subsequently resected. Histopathology showed mixed type of epithelioid and spindle cells with exon 11 KIT mutations. Early diagnosis, prompt management, and long-term followup are the key prognostic determinants.
\end{abstract}

Keywords: Gastrointestinal stromal tumor; GIST; KIT mutation; Irregular gastric lump; Melena

\section{Introduction}

Gastrointestinal stromal tumors in the pediatric age group are very rare and involve teenage girls in $85 \%$ of cases [1]. Immunocytochemistry and mutational analysis has revolutionized the diagnosis of these tumors. Although GIST is a rare cause of GI bleeding, it should be considered as differential diagnosis in cases of hematemesis, melena, unexplained anemia, and weight loss. Treatment is by surgical resection with tyrosine kinase inhibitors demonstrating promising results in metastatic, non-resectable, and recurrent disease. These patients require frequent and long-term surveillance.

\footnotetext{
Manuscript accepted for publication September 11, 2013

${ }^{\text {a} D e p a r t m e n t ~ o f ~ P a e d i a t r i c ~ S u r g e r y, ~ R o y a l ~ M a n c h e s t e r ~ C h i l d r e n ~}$ Hospital, Manchester, UK

${ }^{\mathrm{b}}$ Department of Gastroenterology, Royal Manchester Children Hospital, Manchester, UK

${ }^{c}$ Department of Radiology, Colchester General Hospital, Colchester, CO4 5JL, UK

${ }^{\mathrm{d}}$ Corresponding author: Shagufta Jabeen, Department of Paediatric Surgery, Royal Manchester Children Hospital, Manchester, M13 9WL, UK. Email: sheenjeem1@gmail.com
}

doi: http://dx.doi.org/10.4021/jmc1491w

\section{Case Report}

A 13-year-old British white girl was referred from a district general hospital with history of two episodes of dizziness, chest pain, collapse, and melena in over a 4-week period requiring transfusion. She was commenced on omeprazole and sucralfate on the presumption that a peptic ulcer was the cause of the upper gastrointestinal (GI) bleeding. Upper GI endoscopy revealed a mass in the body and antrum of the stomach with erosive ulceration. Endoscopic biopsy results showed chronic mild gastritis (Fig. 1a). Abdominal ultrasound (Fig. 1b) and contrast-enhanced computerized tomography (CECT) revealed irregular mural mass of discrete lumps (Fig. 2a) with necrotic areas, projecting from anterior wall of the distended stomach into the body and antrum.

Laparoscopic biopsy (Fig. 2b) was performed to ascertain the diagnosis before surgical intervention. Mutational screening displayed a mutation in exon 11 of tyrosineprotein kinase (KIT) which resulted in an in-frame deletion and had been previously reported in gastrointestinal stromal tumor (GIST) [2].

At the time of definitive surgery, a large multinodular mass measuring $13 \times 7 \times 3.5 \mathrm{~cm}$ was identified encompassing the body and antrum of the stomach. Partial gastrectomy with Roux-en-Y gastrojejunostomy was performed. The tumor was positive for CD117 and DOG1 and showed mixed spindle and epithelioid cells with mitotic count of $5 / 50 \mathrm{~mm}^{2}$. The mutational analysis confirmed mutation in exon 11 of KIT. A small peritoneal deposit taken from mid jejunum for histopathology confirmed metastatic GIST. The patient had an uneventful post-operative period and was discharged home on three months follow-up full blood count test to review her anemia status and $\mathrm{CT}$ abdomen to detect recurrence.

\section{Discussion}

GIST is a rare mesenchymal neoplasm and represents 2-3\% of the gastrointestinal tract tumors [3]. It has bimodal age distribution, commonly found in middle aged patients with equal gender distribution [4]. The rarer peak is seen in teen- 


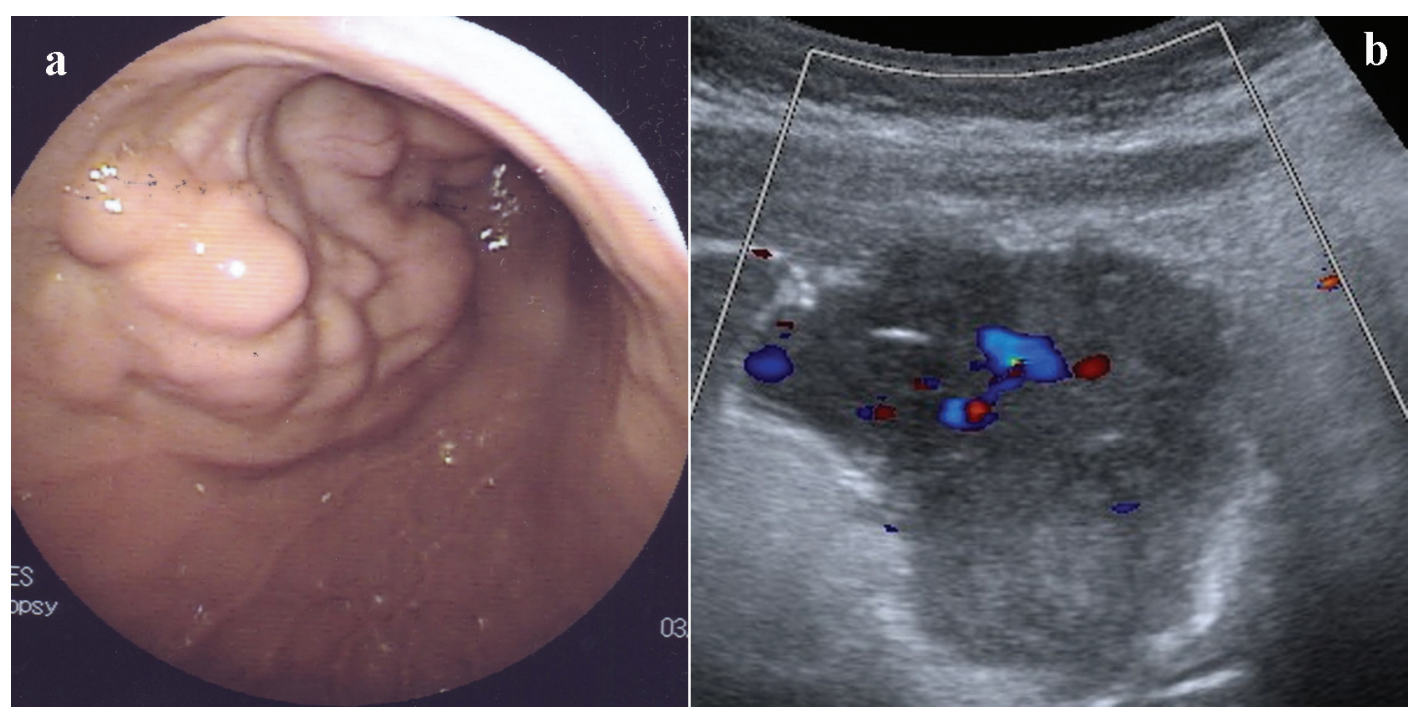

Figure 1. (a) Endoscopic view of GIST. (b) Ultrasound showing irregular heterogenous epigastric mass.

ager girls, 2.7 folds more often as compared to boys. Median age of presentation is 12.4 years in children [5]. Few congenital cases of the GISTs have also been reported in literature [6]. Stomach is the most common site for GIST $(47-60 \%)$ with other sites of involvement in descending order being small bowel (30\%) [7], large bowel (8-10\%), and esophagus $(2 \%)$. Rarely peritoneum is the primary site [8].

GISTs arise from interstitial cells of Cajal (ICC) "the pacemaker cells" of muscularis propria of the gastrointestinal tract as a result of a mutation in KIT in $75 \%$ or platelet derived growth factor receptor alpha (PDGFRA) at 4q12 locus in $8-11 \%$ of adult cases [1]. There are a number of other mutations that have also been identified. Eighty-five percent of pediatric GISTs lack KIT or PDGFRA gene mutations and are categorized as wild-type (WT GIST) [9]. This compares to only $15 \%$ of adult GIST being wild type. Oncogenesis in this type of GIST is unknown and this variety of tumor is less sensitive to tyrosine kinase inhibitors such as Imatinib [10]. GISTs are divided into three histological groups: epithelioid cell type, common in girls, spindle cell type, prevalent in boys, and mixed cell type affecting both sexes equally. Macroscopically tumor is usually a round or multilocular mass.

Pediatric GISTs can be sporadic without any syndromic association; familial, for example Carney-Stratakis syndrome; or a part of non-hereditary Carney's triad. It has been postulated that some children with a GIST may represent the initial presentation of a forme fruste of Carney's triad [11] who then develop another tumor of this triad (extra-adrenal paraganglioma and pulmonary chondroma) later in life. Thus, the time between the initial presentation of the GIST
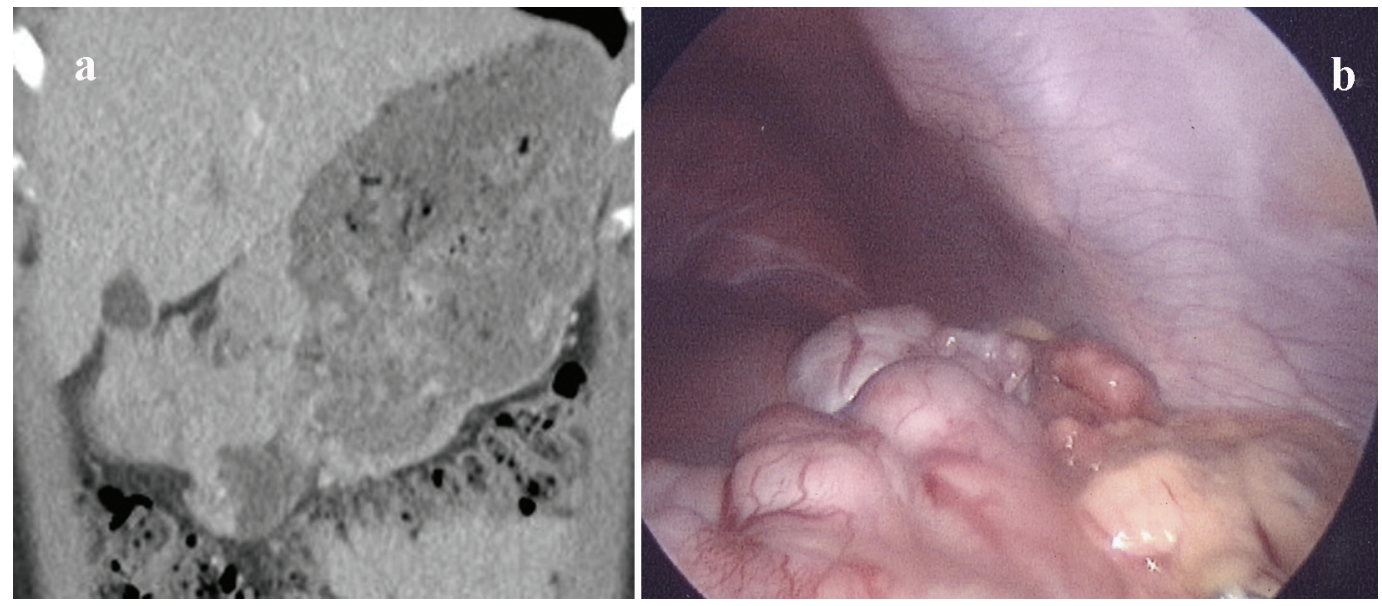

Figure 2. (a) CECT showing multilocular solid mass with central necrosis in a nodule. (b) Laparoscopic view of anterior wall of stomach showing multilocular GIST. 
and the other tumors of Carney's triad may vary greatly, even decades.

Clinical presentation of GIST varies greatly ranging from vague symptoms to upper GI bleeding or obstruction. Approximately $50 \%$ patients present with melena or hematemesis, $20-40 \%$ present with abdominal pain and $20 \%$ with obstruction secondary to abdominal mass [4]. Early satiety, indigestion, and bloating are other clinical features.

Ultrasound, CT, positron emission tomography (PET), and PET-CT scanning have been used to diagnose GIST, its treatment response and follow-up [12]. In adults, following radiological diagnosis, primary resection is performed without initial biopsy as laparoscopic biopsy can increase subsequent risk of pseudo-capsule rupture and peritoneal seeding. In our patient we were concerned that as lymphoma may present with a similar picture biopsy was imperative in directing appropriate management.

Following investigation, surgical resection is dictated by the site of the tumor and may include partial/total gastrectomy, wide local resection of primary tumor, and end-to-end anastomosis. Emergency surgical resection is warranted as a primary procedure in acute uncontrollable or ongoing hemorrhage to relieve obstruction.

Generally, GISTs are resistant to conventional chemotherapy and radiotherapy. Imatinib and newer tyrosine kinase inhibitors have successfully been used for medical management of malignant or recurrent disease and debulking before resection [13]. Risk assessment is based upon tumor size and mitotic count [14] and is vital for follow-up strategy as given below:

1) Very low risk (size less than $2 \mathrm{~cm}$, mitotic count less than $5 / 50$ high power field HPF): No follow-up imaging is required

2) Low risk (size $2-5 \mathrm{~cm}$, mitotic count less than $5 / 50$ HPF): CT at 3 months after resection then clinical follow-up

3) Intermediate risk (size less than $5 \mathrm{~cm}$, count 6-10 HPF or size $5-10 \mathrm{~cm}$, count less than 5/50 HPF): CT at 3 months post resection, followed by every 6 months for 2 years, then annually up to 5 years

4) High risk (size greater than $5 \mathrm{~cm}$, count more than $5 / 50 \mathrm{HPF}$ or size 10 irrespective of count or count more than 10/50 PHF irrespective of size): CT every 3 months for 2 years, followed by 6 months for 2 years and then annually.

Recurrence is directly proportional to the risk group. Pediatric GISTs are more likely to recur at their primary site as compared to metastasizing. The multi-focal character of pediatric gastric tumor is also thought to be responsible for local recurrence. GIST can metastasize to peritoneum, liver, lungs, and bones. Lymph node metastasis has been found mostly in pediatric cases [15]. Overall 5-year survival rate has been reported to be $35 \%$ [4].

Our case is exceptional as sporadic gastric tumors are very rare in this age group as is exon 11 KIT mutation with synchronous peritoneal metastasis. This mutation is usually found in Carney's triad, therefore it could be forme fruste Carney's triad. Because of the chances of metachronous tumor, recurrence and metastasis long-term follow-up is mandatory.

\section{Conflict of Interest}

None.

\section{References}

1. Liegl-Atzwanger B, Fletcher JA, Fletcher CD. Gastrointestinal stromal tumors. Virchows Arch. 2010;456(2):111-127.

2. Lasota J, Jasinski M, Sarlomo-Rikala M, Miettinen M. Mutations in exon 11 of c-Kit occur preferentially in malignant versus benign gastrointestinal stromal tumors and do not occur in leiomyomas or leiomyosarcomas. Am J Pathol. 1999;154(1):53-60.

3. Manoj K, Rajiv J, Sangram S, et al. Leiomyoma of the mesentery in an infant. Journal of Indian Association of Pediatric Surgeons. 2007;12:142-144.

4. Abbassi-Ghadi N, Bartlam K, Rasheed S, Holme T. A case of obscure gastrointestinal bleeding in a teenager. Ann R Coll Surg Engl. 2009;91(5):W1-3.

5. Kaemmer DA, Otto J, Lassay L, Steinau G, Klink C, Junge $\mathrm{K}$, Klinge $\mathrm{U}$, et al. The Gist of literature on pediatric GIST: review of clinical presentation. J Pediatr Hematol Oncol. 2009;31(2):108-112.

6. Geramizadeh B, Bahador A, Ganjei-Azar P, Asadi A. Neonatal gastrointestinal stromal tumor. Report of a case and review of literature. J Pediatr Surg. 2005;40(3):572574.

7. Choti MA. Gastric Gastrointestinal Stromal Tumors. 2013; retrieved from: http://emedicine.medscape.com/ article/278845-overview.

8. Dahnert W. Radiology Review Manual 6th ed. 2007;837.

9. Janeway KA, Kim SY, Lodish M, Nose V, Rustin P, Gaal J, Dahia PL, et al. Defects in succinate dehydrogenase in gastrointestinal stromal tumors lacking KIT and PDGFRA mutations. Proc Natl Acad Sci U S A. 2011;108(1):314-318.

10. Sleijfer S, Wiemer E, Seynaeve C, Verweij J. Improved insight into resistance mechanisms to imatinib in gastrointestinal stromal tumors: a basis for novel approaches and individualization of treatment. Oncologist. 2007;12(6):719-726.

11. Hayat H, Gilbey S G. Carney's Triad, Endocrine Abstracts. 2007;13;101.

12. Reid R, O'Dywer P, MacDuff E, et al. Guidelines for the Management of Gastrointestinal Stromal Tumours (GIST) In Scotland. 2000;20-23. 
13. Jiang Y, Ming L, Montero AJ, Kimchi E, Nikfarjam M, Staveley-O'Carroll KF. Optimizing imatinib mesylate treatment in gastrointestinal stromal tumors. Gastrointest Cancer Res. 2008;2(5):245-250.

14. Shirin H, Kravtsov V, Shahmurov M, Shabat VS, Krinshpon I, Alin A, Avinoach I, et al. The cyclin-dependent kinase inhibitor, p27, has no correlation with the malig- nant potential of GIST. Digestion. 2007;75(1):4-9.

15. Otto C, Agaimy A, Braun A, Radecke J, Hoeppner J, Illerhaus G, Werner M, et al. Multifocal gastric gastrointestinal stromal tumors (GISTs) with lymph node metastases in children and young adults: a comparative clinical and histomorphological study of three cases including a new case of Carney triad. Diagn Pathol. 2011;6:52. 